\title{
Experimental Verification of two Theoretical Solutions of the Pendulum for Large Angles in Frequency Domain for Teaching Support
}

\author{
https://doi.org/10.3991/ijim.v14i08.12607 \\ Y. A. Garcés-Gómez ${ }^{(\varpi)}$, P. A. López, O. O. Cárdenas, V. Henao-Cespedes \\ Universidad Católica de Manizales, Manizales, Colombia \\ ygarces@ucm.edu.co \\ N. Toro-García \\ Universidad Nacional de Colombia, Sede Manizales, Colombia
}

\begin{abstract}
During pendulum analysis, the approximation for small angles is usually performed as a simple harmonic motion. However, for large angles this approximation is not convenient so exact solutions are proposed by different methods. This paper presents the comparison of two solutions for the displacement of the pendulum in the domain of time and frequency, the solution by Jacobi elliptic function and the solution by the numerical method Dormand-Prince with the results of measurements obtained by means of a physical prototype designed for the teaching of physics. We consider that this comparative study allows a better understanding of the phenomenon of the non-linear pendulum in the students of undergraduate careers in the physics of waves as well as a previous training for the course of analysis of signals being in good exercise of teaching.
\end{abstract}

Keywords - Technology-enhanced learning, Pendulum, frequency domain, exact solution, Fourier analysis, numerical methods

\section{Introduction}

Technology-enhanced Learning (TEL), which is based on the advance of information and communication technologies, allows students to acquire a deeper knowledge of the phenomena and concepts studied. On the other hand, it is also clear that new generations feel very comfortable with intelligent mobile devices and it is undeniable that these technologies have permeated the classroom because a technology-enhanced environment offers students the opportunity to design and implement experiments, explore the relationships between variables and thus learn scientific concepts $[1,2]$.

The teaching of physical phenomena has been strongly permeated by technologies, from laboratory instrumentation systems, remote access to physical laboratories, virtual environments for experimentation and intelligent mobile devices [3-6] which has allowed an important advance in the didactics of learning [7]. 
One of the non-linear systems widely studied in both introductory and advanced mechanical physics courses is the simple pendulum [8-15]. The differential equation that models the simple undamped pendulum is given by (1):

$$
\frac{d^{2} \theta}{d t^{2}}+\omega_{0}^{2} \sin \theta=0
$$

with $\omega_{0}=\sqrt{\frac{g}{l}}$.

Different types of solutions to the equation (1) have been defined. In [16] it is proposed that the angular displacement $\theta$ of a mass $\mu$ oscillating in a vertical plane supported by a massless string of length $l$ described by the non-linear differential equation (1), has a solution through Jacobian Amplitude Function $\operatorname{am}(x, m)$ as (2):

$$
\theta(t)=2 a m\left[\left(\frac{g}{m l}\right)^{\frac{1}{2}} ; m\right]
$$

where $m=\frac{2 \mu g l}{E_{0}}$, and $E_{0}$ is the total mechanical energy, which is constant for the system.

In $[11,17]$ it is argued that the solution for (1) is given by (3) in the following way:

$$
\theta(t)=2 \arcsin \left\{\sin \frac{\theta_{0}}{2} \operatorname{sn}\left[K\left(\sin ^{2} \frac{\theta_{0}}{2}\right)-\omega_{0} t ; \sin ^{2} \frac{\theta_{0}}{2}\right]\right\}
$$

with $\theta_{0}$ that is the initial amplitude and $K$ is the complete elliptical integral of the first kind defined as:

$$
K(m)=\int_{0}^{1} \frac{d z}{\sqrt{\left(1-z^{2}\right)\left(1-m z^{2}\right)}}
$$

In $(3) \operatorname{sn}(u ; m)$ is the Jacobi elliptic function [18].

On the other hand, solutions to differential equations can be found by numerical methods such as the Dormand-Prince $[19,20]$ that can solve problems of initial value from (5).

$$
y_{(n+1)}=y_{n}+h \sum_{i=1}^{s} b_{i} k_{i}
$$

\subsection{Problem of research}

This document discusses the problem of teaching the pendulum movement as a composite harmonic movement for large angles from the frequency frame of reference. Frequency study is proposed because the study of the pendulum for introductory courses is usually done in the time domain where waveforms are studied as simple harmonics given that visually they seem so and that mathematical approximations demonstrate it in the same way. 


\subsection{Research focus}

The research focuses on determining the different forms of solution that exist as reported in a review of the systematic literature, then develops a method of solution by numerical approximations and finally captures data from a physical system. An algorithm is developed that allows to visualize the results in the frequency domain for analysis and comparison finding an important correlation between the results that would not be possible to analyze with the students of the introductory courses with the signals in the time domain.

\section{Numerical Solutions}

Two theoretical solutions have been implemented for the development of this work. The first is developed by means of the exact solution for the pendulum of the equation (3). In this procedure the Jacobi elliptic function is found from the routine of Matlab® as in Figure 1.

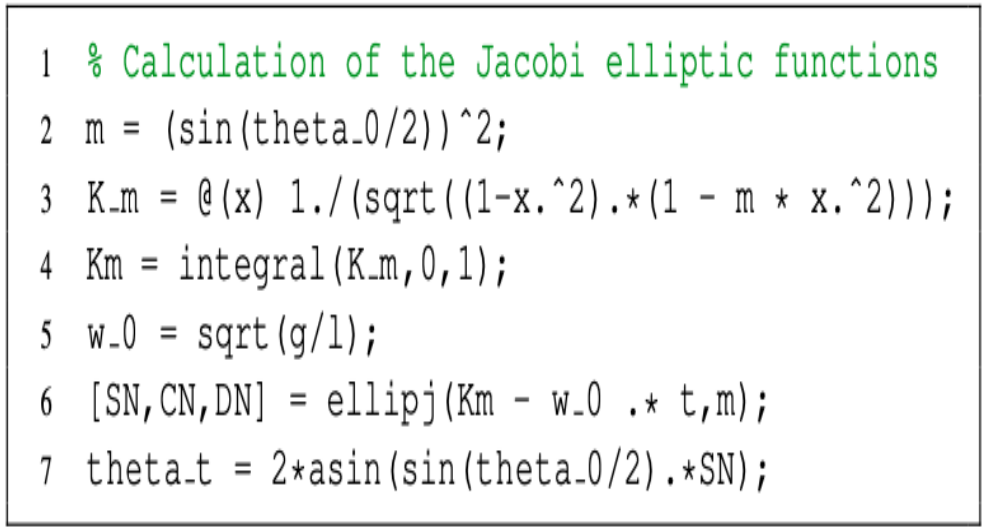

Fig. 1. Algorithm to calculate the Jacobi elliptic functions

The second solution has been proposed by numerical methods using the Matlab® ODE45 package with which the second-degree differential equation has been solved and the solution has been obtained in time for the displacement of the pendulum.

The parameters that have been used for the solution are presented in Table 1

Table 1. Parameters used for solution.

\begin{tabular}{|c|c|}
\hline Parameter & Value \\
\hline$l$ & $0,8 \mathrm{~m}$ \\
\hline$\theta_{0}$ & $\pi / 2 \mathrm{rad}$ \\
\hline$t$ & $9 \mathrm{~s}$ \\
\hline$\Delta t$ & $0,01 \mathrm{~s}$ \\
\hline$g$ & $9,8 \mathrm{~m} / \mathrm{s}^{2}$ \\
\hline
\end{tabular}


For the presentation of the results the Fast Fourier Transform is used [21], in this way it is possible to observe the results in the frequency domain since the variations in the time domain are not perceptible in the graphs found. Single-Sided Amplitude Spectrum is presented.

Fuourier's discrete transform is defined by (6):

$$
Y(k)=\sum_{j=1}^{n} X(j) W_{n}^{(j-1)(k-1)}
$$

\section{Experimental Implementation}

For the capture of experimental data, a prototype of a pendulum used as a didactic tool in wave physics laboratories has been implemented [21]. The prototype is presented in the Fig. 2.

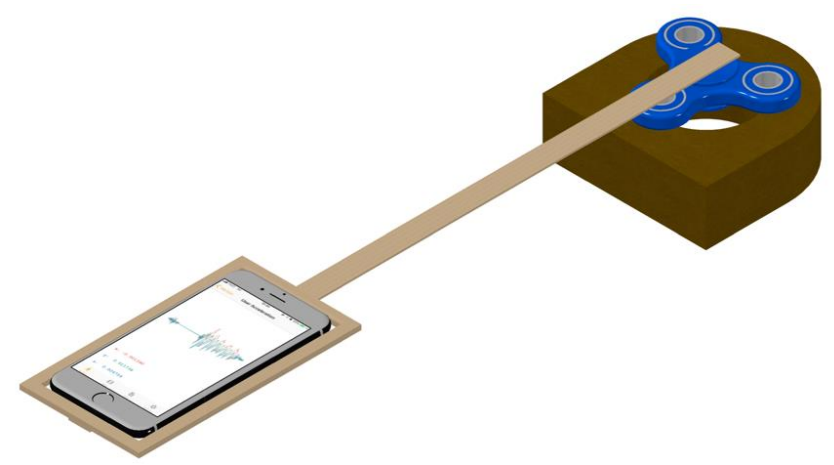

Fig. 2. Experimental prototype developed for physics didactics

The prototype is implemented in a heavy support to fix it in flat surfaces to which a fixed spinner is stuck, from its central axis the bar of despicable mass is fixed and at the end a support for the mobile device own of the student. Data is captured in real time through the application phyphox [22].

\section{$4 \quad$ Results}

In Figure 3 the signals obtained as a function of time are displayed. In the signal obtained with the numerical method is observed that there is an increase in the period of the signal, this increase is because the calculation by the method Dormand-Prince generates a delay to take previous steps to generate subsequent steps. 


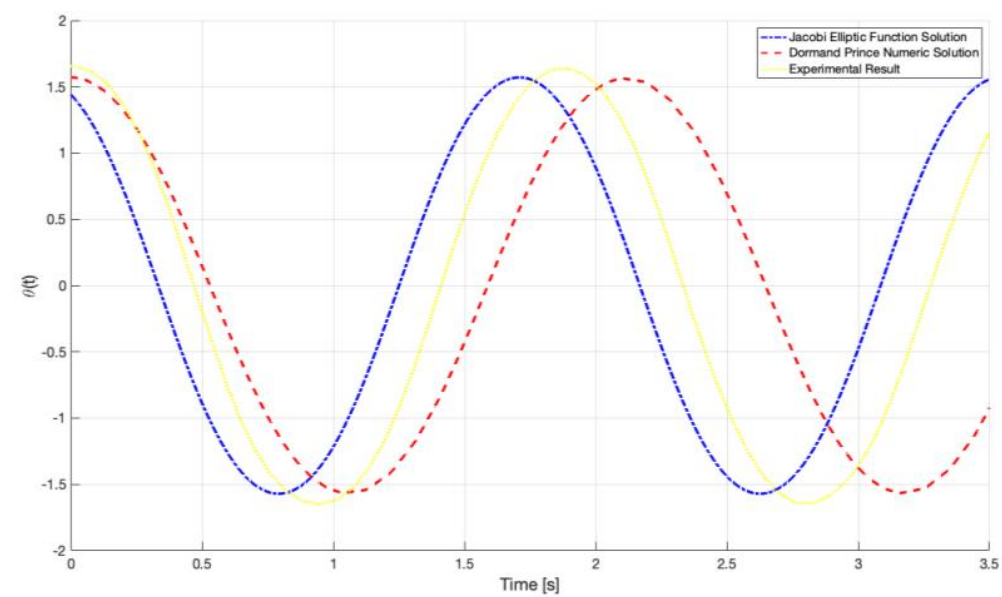

Fig. 3. Experimental prototype developed for physics didactics

Figure 4 shows the frequency analysis of the three signals presented in Figure 3.
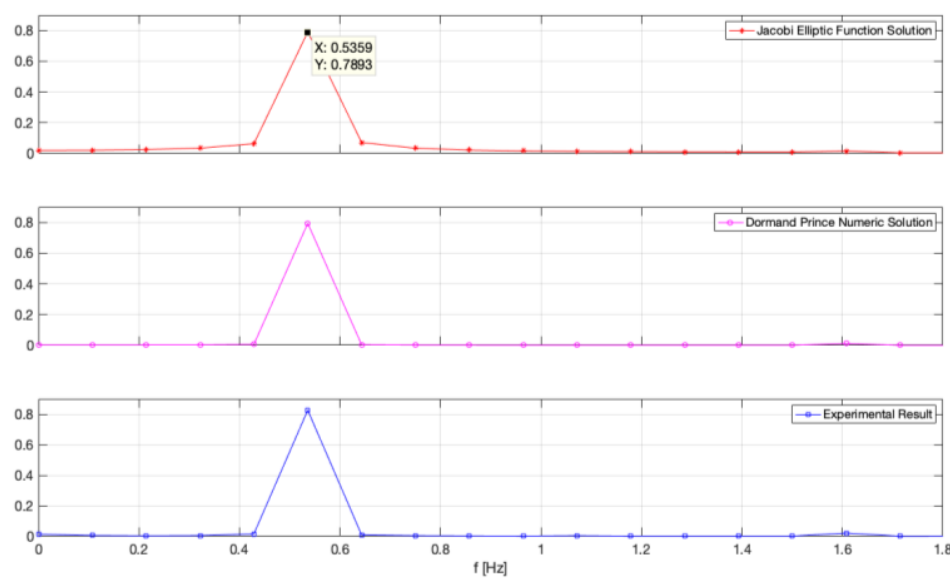

Fig. 4. Time signals

The theoretical period of the system is given by (7) [23].

$$
P=\frac{2 P_{0}}{2} \int_{0}^{\pi / 2} \frac{d \theta}{\sqrt{1-k^{2} \sin ^{2} \theta}}=1,8351
$$

where $P_{0}=2 \pi \sqrt{\frac{l}{g}}$ is the period with small angles. With the above result the fundamental frequency is $f=0,5449 \mathrm{~Hz}$.

In Figure 5 zoom in amplitude to the signals in the frequency to see the 3 th harmonic for the three solution cases. 


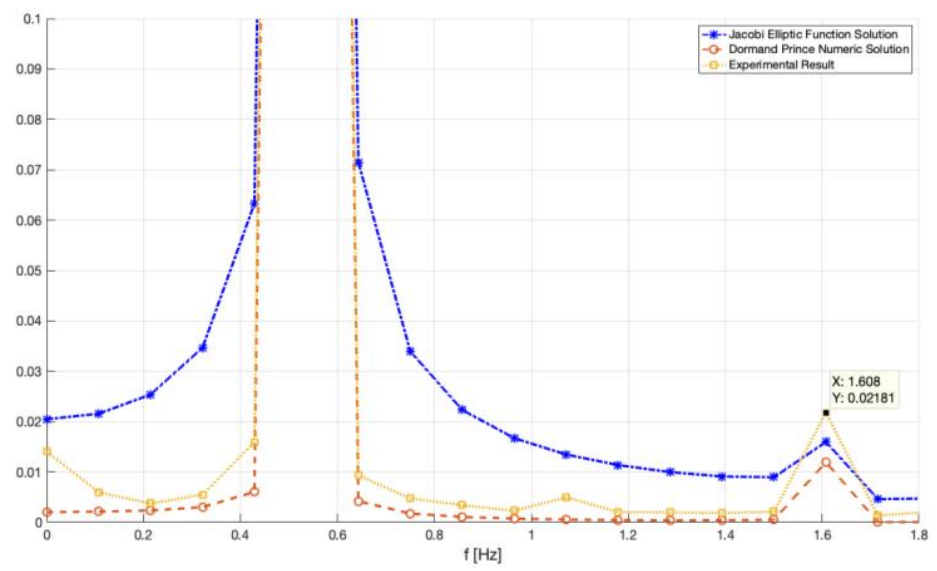

Fig. 5. Comparison of the 3rd harmonic

Figure 6 shows the zoom for the 5th harmonic, the exact solution by the Jacobi Elliptic Function due to the continuous amplitude shows the harmonic in a very reduced form. The experimental measurement shows the highest relative amplitude of the 5th harmonic. Also observed in the numerical solution are the 7th, 9th, and other odd harmonics that are also in the experimental result that are masked by the noise in the signal.

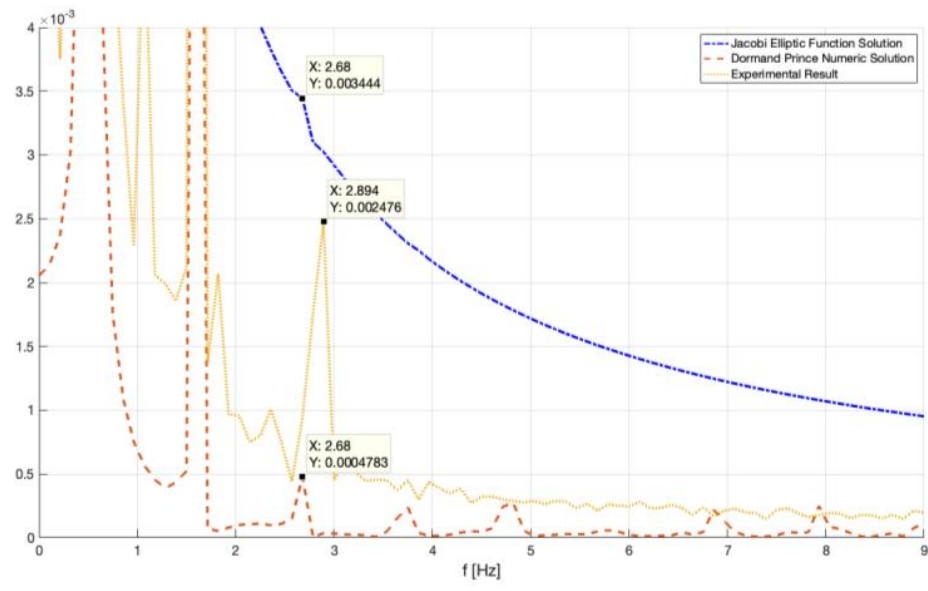

Fig. 6. Comparison of the 5rd harmonic

Frequency components can be shown in the signal by means of the expansion in Taylor series of the function $\sin \frac{\theta_{0}}{2}$ as (8) [13].

$$
\sin \frac{\theta_{0}}{2}=\sum_{n=1}^{\infty} \frac{(-1)^{n+1} \theta_{0}^{2 n-1}}{2^{2 n-1(2 n-1) !}}
$$


In Figure 7 Frequency components are shown in bar format for better comparison.

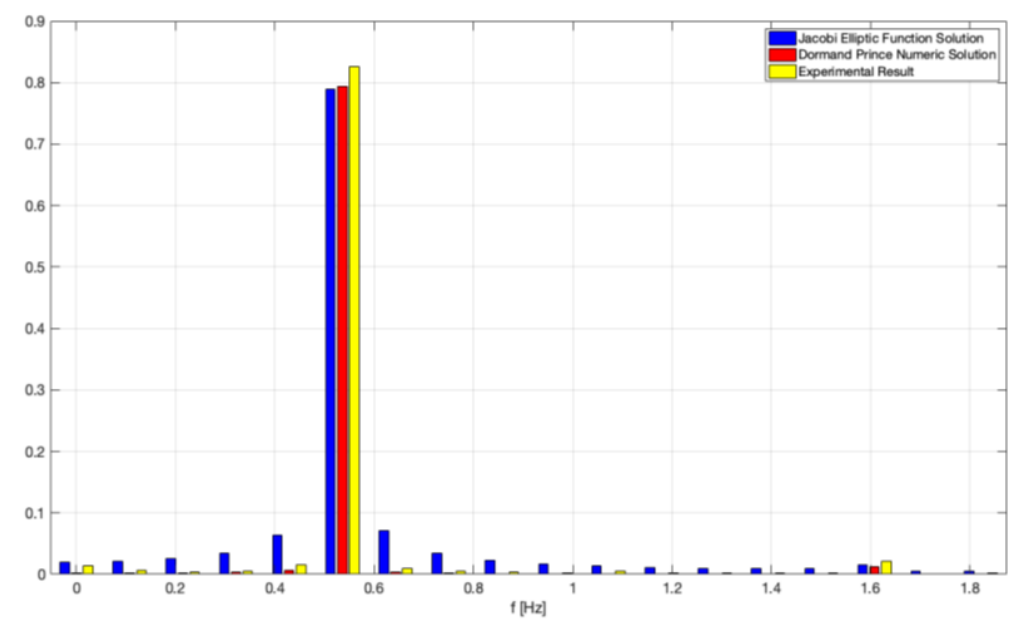

Fig. 7. Bar graph of the first harmonics in three signals

\section{Discussion}

From the results it can be found that for larger angles the analysis of the signal as a pure periodic signal is not intuitive, this is because other components of low amplitude appear that are not perceptible in the periodic wave. From the frequency analysis it was possible to verify that there are harmonics, which for the analyzed case, corresponds to the 3th that appears both in the two mathematical solutions and in the experimental data that can be observed in detail in Fig. 5. There are also odd harmonics of higher frequency that can be demonstrated with the solution of the equation in Taylor series [24]. Analysis in the frequency domain allows a deeper understanding of the phenomenon of pendulum displacement as a harmonic system composed of different odd frequencies. The results show that the graphic analysis in time domain is not suitable to detect the variations in waves, in the other hand, with the frequency analysis students can observe how as the angle of displacement increases, in the frequency appear new harmonic components in wave.

\section{Conclusion}

The study of physics requires an important abstraction and association of the physical phenomenon with the mathematical formulation that describes it. This description is usually strong in formalisms and deep concepts of mathematics that require long and complex developments for students of introductory courses in physics. In order to simplify the developments, approximations are usually made in developments such as the case of the pendulum for small angles as a simple harmonic movement given its 
similarity with the uniform circular movement. However, approximations can induce students to conceptual errors or to consider that the physical phenomenon always has the same behavior under all conditions leading to not associating the phenomenon correctly with the mathematical formulation.

Based on the above, it is extremely necessary to look for other tools that allow a better understanding of the phenomenon and for undulatory phenomena, analysis in the frequency can be a very good visual approximation to the deep reality of the phenomenon analyzed.

\section{$7 \quad$ Acknowledgement}

The author thanks Universidad Católica de Manizales with the Research Group on Technological and Environmental Development GIDTA and Education and Educators Traning EFE and the Universidad Nacional de Colombia - Sede Manizales.

\section{$8 \quad$ References}

[1] E.Y. Feyzioğlu, E. Akpinar, N. Tatar, Effects of technology-enhanced metacongnitive learning platform on students' monitoring accuracy and undestanding of electricity, J. Ob Balt. Sci. Educ. 17 (2018) 43-64. http://oaji.net/articles/2017/987-1519059947.pdf.

[2] H.M. Al Fawareh, S. Jusoh, the use and effects of smartphones in higher education, Int. J. Interact. Mob. Technol. 11 (2017) 103-111.https://doi.org/10.3991/ijim.v11i6.7453

[3] F. Bertacchini, E. Bilotta, F. Caldarola, P. Pantano, the role of computer simulations in learning analytic mechanics towards chaos theory: a course experimentation, Int. J. Math. Educ. Sci. Technol. 50 (2019) 100-120. https://doi.org/10.1080/0020739x.2018.1478134.

[4] J.A. Frank, V. Kapila, Mixed-reality learning environments: Integrating mobile interfaces with laboratory test-beds, Comput. Educ. 110 (2017) 88-104. https://doi.org/10.1016/ j.compedu.2017.02.009.

[5] H. Crompton, B. Olszewski, T. Bielefeldt, The mobile learning training needs of educators in technology-enabled environments, Prof. Dev. Educ. 42 (2016) 482501.https://doi.org/10.1080/19415257.2014.1001033.

[6] N.D. Finkelstein, W.K. Adams, C.J. Keller, P.B. Kohl, K.K. Perkins, N.S. Podolefsky, S. Reid, R. Lemaster, when learning about the real world is better done virtually: A study of substituting computer simulations for laboratory equipment, Phys. Rev. Spec. Top. - Phys. Educ. Res. 1 (2005) 1-8. https://doi.org/10.1103/physrevstper.1.010103.

[7] M. Alwraikat, Smartphones as a new paradigm in higher education overcoming obstacles, Int. J. Interact. Mob. Technol. 11 (2017) 114-135. https://doi.org/10.3991/ijim.v11 i4.6759.

[8] B.F. Davis, L.P. Fulcher, Theoretical and experimental study of the motion of the simple pendulum, Am. J. Phys. 44 (1976) 51-55. https://doi.org/doi.org/10.1119/1.10137 View.

[9] R.A. Nelson, M.G. Olsson, The pendulum-Rich physics from a simple system, Am. J. Phys. 54 (1986) 112-121. https://doi.org/10.1119/1.14703.

[10] G.L. Baker, J.A. Blackburn, The ppendulum, a case study in physics, Oxford University Press, New York, 2005. https://doi.org/10.1017/CBO9781107415324.004. 
[11] A. Beléndez, C. Pascual, D.I. Méndez, T. Belénez, C. Neipp, Exact solution for the nonlinear pendulum, Rev. Bras. Ensino Física. 29 (2007) 645-648. https://doi.org/10.1590/ s1806-11172007000400024

[12] F.M.S. Lima, Analytical study of the critical behavior of the nonlinear pendulum, Am. J. Phys. 78 (2010) 1146-1151. https://doi.org/10.1119/1.3442472.

[13] D.G. Simpson, The nonlinear pendulum, Dep. Phys. Sci. Eng. Dec (2010) 5.

[14] K. Johannessen, An anharmonic solution to the equation of motion for the simple pendulum, Eur. J. Phys. 32 (2011) 407-417.https://doi.org/10.1088/0143-0807/32/2/014

[15] V. V. Mayer, E.I. Varaksina, An apparatus to demonstrate linear and nonlinear oscillations of a pendulum, Phys. Educ. 51 (2016) 45012.https://doi.org/10.1088/0031$\underline{9120 / 51 / 4 / 045012}$

[16] K.L. Sala, Transformations of the Jacobian Amplitude Function and its Calculation Via the Arithmetic-Geometric Mean*, Soc. Ind. Appl. Math. SIAM J. Math. Anal. 20 (1989) 1514-1528. https://doi.org/10.1137/0520100

[17] F.W.J. Olver, D.W. Lozier, F. Boisvert, Ronald, C.W. Clark, NIST Handbook of Mathematical Functions, National Institute of Standards and Technology and Cambridge University Press, United States of America, 2010.

[18] A.H. Khater, M.M. Hassan, R.S. Temsah, Exact solutions with Jacobi elliptic functions of two nonlinear models for ion-acoustic plasma waves, J. Phys. Soc. Japan. 74 (2005) 14311435. https://doi.org/10.1143/ipsj.74.1431

[19] J.R. Dormand, P.J. Prince, A family of embedded Runge-Kutta formulae, Comput. Appl. Math. 6 (1980).

[20] L.F. Shampine, M.W. Reichelt, The matlab ode suit, (n.d.) 1-22.

[21] L. Martínez, P. Garaizar, Learning Physics Down a Slide: A Set of Experiments to Measure Reality Through Smartphone Sensors, Int. J. Interact. Mob. Technol. 8 (2014) 40.https://doi.org/10.3991/ijim.v8i3.3873

[22] S. Staacks, S. Hütz, H. Heinke, C. Stampfer, Advanced tools for smartphone- based experiments: phyphox, Phys. Educ. 53 (2018) 6. https://doi.org/10.1088/1361-6552/aac05e

[23] W.H. Press, S.A. Teukolsky, W.T. Vetterling, B.P. Flannery, Numerical recipes in C. Vol. 2, Cambridge: Cambridgeuniversity press, 1992.

[24] Y. Yamashita, Foucault Pendulum without Driving, Ournalof Phys. Soc. Japan. 85 (2016) 064002. https://doi.org/10.7566/jpsj.85.064002

\section{Authors}

Eason Alberto Garcés-Gómez is member of the Academic Training Unit in Natural Sciences and Mathematics at Universidad Católica de Manizales, Cra 23 No 6063, Manizales, Colombia. He works as researcher in didactics of sciences and applied technologies to education and healt in the Research Groups in Technological and Environmental Development and Educational and Educators Traning. He has a Master and a Ph.D. Degrees in Engineering. Email: ygarces@ucm.edu.co

Paula Andrea López Jimenez is the director of the Academic Training Unit in Natural Sciences and Mathematics at Universidad Católica de Manizales. She works as researcher in didactics of sciences and has a Master Degree in sciences teaching.

Oscar Oswaldo Cárdenas Delgado is member of the Academic Training Unit in Natural Sciences and Mathematics at Universidad Católica de Manizales. He works as researcher in didactics of sciences and has a Master Degree in maths didactics. 
Vladimir Henao-Cespedes is member of the Academic Training Unit in Natural Sciences and Mathematics at Universidad Católica de Manizales. He works as researcher in applied technologic developments in the Research Group on Technological and Environmental Development. He has a Master and Ph.D. Degree in engineering.

Nicolás Toro-García is member of the Electrical and Electronics Department at Universidad Nacional de Colombia - Sede Manizales. He works as researcher and has a Ph.D. degree in Engineering.

Article submitted 2019-12-03. Resubmitted 2020-02-25. Final acceptance 2020-02-26. Final version published as submitted by the authors. 